\title{
Electrophoretic mobility of a charged colloidal particle: A computer simulation study
}

\author{
Vladimir Lobaskin $\dagger$, Burkhard Dünweg, and Christian Holm \\ Max Planck Institute for Polymer Research, Ackermannweg 10, D-55128 Mainz, \\ Germany
}

\begin{abstract}
We study the mobility of a charged colloidal particle in a constant homogeneous electric field by means of computer simulations. The simulation method combines a lattice Boltzmann scheme for the fluid with standard Langevin dynamics for the colloidal particle, which is built up from a net of bonded particles forming the surface of the colloid. The coupling between the two subsystems is introduced via friction forces. In addition explicit counterions, also coupled to the fluid, are present. We observe a non-monotonous dependence of the electrophoretic mobility on the bare colloidal charge. At low surface charge density we observe a linear increase of the mobility with bare charge, whereas at higher charges, where more than half of the ions are co-moving with the colloid, the mobility decreases with increasing bare charge.

PACS numbers: 82.45.+z,82.70.Dd,66.10.-x,66.20.+d
\end{abstract}

Submitted to: J. Phys.: Condens. Matter

$\dagger$ To whom correspondence should be addressed (lobaskin@mpip-mainz.mpg.de) 


\section{Introduction}

Many important properties of colloidal dispersions are directly or indirectly determined by the electric charge of the colloidal particles. The charge and the associated electrostatic potential normally yield a repulsive interaction between the particles that stabilizes the dispersion. The current understanding of electro-rheological phenomena is based on a central quantity, the electrokinetic potential or zeta $(\zeta)$ potential, which is decisive for e. $\mathrm{g}$. the electrophoretic motion of the particles, the overall flow behavior of the dispersion, sedimentation, and flocculation. Calculating both the electrostatic and the electrokinetic potential, and establishing a relation between them, is however a challenging task, which has attracted the attention of colloid scientists for decades. ${ }^{1-4}$ For the electrokinetic potential, the main obstacle is the strong coupling between the electric and hydrodynamic degrees of freedom. Partial decoupling is only possible in the case of weak external fields or for unperturbed double layers.

In a constant and homogeneous electric field a charged particle accelerates until the friction force, which is proportional to the velocity, equals the electrostatic force. The electrophoretic mobility $\mu$ of a charged particle is defined as the ratio between its stationary velocity $v_{c}$ and the applied electric field $E$,

$$
\mu=v_{c} / E \text {. }
$$

In the classical theories, it is assumed that one can define a so-called shear plane (or slip surface), which divides the surrounding fluid into two parts: The inner part that moves with the particle, due to strong electrostatic and hydrodynamic coupling of the electric double layer to the colloidal surface, and the outer part which typically moves in the opposite direction, due to the current of the counterions. The zeta potential is defined as the electrostatic potential at the slip surface, and therefore in general differs from the potential at the particle surface. Once this potential is known, the remaining calculation becomes more or less straightforward, if still spherical symmetry is assumed. The electrophoretic mobility $\mu$ can be expressed in general through the $\zeta$ potential as

$$
\mu=\frac{\varepsilon \zeta}{6 \pi \eta} f(\kappa R)
$$

where $\eta$ is the solvent viscosity, $\varepsilon=4 \pi \varepsilon_{0} \varepsilon_{r}$ is the dielectric constant of the fluid, composed of the vacuum and relative dielectric constant, $\varepsilon_{0}$ and $\varepsilon_{r}$, respectively, $\kappa^{-1}$ is the Debye screening length, $R$ denotes the colloidal radius, and $f$ is a function of the salt concentration and colloidal radius. For small colloids and / or low electrolyte concentrations $\kappa R \rightarrow 0$ and $f(\kappa R) \rightarrow 1$, and the Hückel-Onsager limit is recovered, ${ }^{5}$ whereas for $\kappa R \rightarrow \infty$ one obtains $f(\kappa R) \rightarrow 3 / 2$, which is known as the HelmholtzSmoluchowski limit. At intermediate values of $\kappa R$, the values of $f(\kappa R)$ depend on the applied theory. ${ }^{6-9}$

The electrophoretic mobility is easily accessible in experiment and represents a valuable source of information for a colloid scientist. ${ }^{3,4}$ The desired microscopic information about the actual size of the particles and their surface potential is however 
not straightforward to extract. The main reason is that the experimental data only provide information about the motion of the particles together with their double layer. The structure of the latter, however, is not easy to calculate analytically, due to the strong electrostatic coupling and the influence of hydrodynamics, such that linear theories will not work, while different analytical approximation schemes give different answers.

A computer simulation including both electric charges and hydrodynamic interactions provides a unique chance to examine the double layer structure and various dynamic properties such as the particle mobility or the conductivity at the same time. There has been a recent Molecular Dynamics (MD) simulation study where the solvent particles were modeled explicitly. ${ }^{10}$ However the authors were more interested in the overcharging effects occurring at high Coulomb coupling. On the other hand, a significant effort had been made to include hydrodynamic effects in a colloidal simulation on a less expensive level, namely via the lattice Boltzmann (LB) method. ${ }^{11-14}$ This latter approach models the colloidal particles as extended hollow spheres, while stick boundary conditions at the surface are implemented via bounce-back collision rules. The method was extended to charged colloids by Horbach and Frenkel. ${ }^{14}$ In their work, the small ions were represented by LB populations, such that the electrostatics is essentially taken into account on the level of the Poisson-Boltzmann equation. Unfortunately, this method has two disadvantages we would like to avoid: Firstly, the discrete nature of the ions and correlations beyond the Poisson-Boltzmann level are not taken into account; secondly, one cannot avoid a leakage of charge into (and out of) the sphere, such that it is hard (if not impossible) to maintain a well-defined Debye layer of charges around it. ${ }^{15}$

For these reasons, we rather prefer to simulate charge-stabilized colloidal dispersions with explicit counterions; this automatically eliminates both problems. The disadvantage (with which we have to live) is however that only a limited range of size ratios between colloidal particle and counterions is accessible. Recently we proposed a suitable hybrid model which implements this philosophy, while keeping the successful representation of hydrodynamics in terms of the LB approach. ${ }^{16}$ The solvent is modeled via $\mathrm{LB}$, while $\mathrm{MD}$ is done for the solute (colloidal particles and counterions). However, in contrast to the bounce-back implementation of the coupling, we rather use point particles ("monomers"). It should be noted that this term refers only to the coupling to the solvent, while the monomers do have a finite size with respect to their mutual interaction. The coupling to the solvent is inherently dissipative in nature: Each monomer is assigned a phenomenological friction coefficient, and the friction force between solvent and particle is proportional to the relative velocity. The flow velocity at the location of the monomer is obtained via linear interpolation from the surrounding lattice sites, and this implies that the lattice spacing should be of the order of the monomer size. The advantage of this approach is that it is quite flexible, because various large objects of soft matter physics (colloidal particles, polymer chains, membranes, etc.) can be built up from elementary monomers, without major restructuring of the underlying simulation program. However, it is not possible to model the colloidal particle 
just in terms of a single monomer. While the electrostatic interactions plus the excluded volume between colloidal particle and counterions could be easily represented in terms of a single strongly charged particle with large repulsion radius, such a particle would have inappropriate hydrodynamic properties. The fluid would be coupled only to the center of the particle, while a faithful representation of the hydrodynamics requires stick boundaries at the surface of the particle, or a good approximation thereof. Furthermore, we wish to faithfully represent the particle's rotational motion. For these reasons, we add a two-dimensional tethered network of monomers which we wrap around the surface of the central sphere, such that the overall structure resembles a raspberry. The coupling to the solvent is then done only via the surface sites in terms of the monomer friction coefficient. For reasonably large friction, this is an excellent approximation to a stick boundary condition, as has been shown in Ref. ${ }^{16}$ in terms of both the translational and the rotational motion of the sphere.

In the present work, we analyze basic electrokinetic properties of such a model colloid, where we restrict ourselves to the case of a single sphere, and a rather elementary analysis. The remainder of this article is organized as follows: In Sec. 2, we describe our simulation model, while Sec. 3 contains the numerical results on the double layer structure and particle mobility in a constant electric field. Finally, Sec. 4 concludes with a brief summary.

\section{Model}

Our hybrid simulation method involves two subsystems: the solvent that is modeled via LB with fluctuating stress tensor (i. e. we run a constant-temperature version of the LB method) and a Langevin MD simulation for the particles immersed in the solvent. The LB simulation is performed using the 18 -velocity model, ${ }^{17}$ while the solute is coupled dissipatively to the solvent as described in. ${ }^{18,19}$ The fluid simulation consists of collision and propagation steps, the former being performed with inclusion of the momentum transfer from the solute particles (surface beads and ions).

The colloidal particle is represented by a two-dimensional tethered bead-spring network consisting of 100 beads, which is wrapped around a ball of a radius $\sigma_{c s}$ (for notation, see below), so that the whole construction resembles a raspberry (see Fig. 1). The network connectivity is maintained via finitely extendible nonlinear elastic (FENE) springs,

$$
V_{F E N E}(r)=-\frac{k R_{0}^{2}}{2} \ln \left(1-\left(\frac{r}{R_{0}}\right)^{2}\right),
$$

where $k$ is the spring constant chosen to be $k=25$, and $R_{0}$ denotes the maximum bond extension, here 1.25. Furthermore, the beads repel each other by a modified LJ potential

$$
V_{L J}(r)= \begin{cases}4 \epsilon_{i j}\left(\left(\frac{\sigma_{i j}}{r}\right)^{12}-\left(\frac{\sigma_{i j}}{r}\right)^{6}+\frac{1}{4}\right) & r<2^{1 / 6} \sigma_{i j} \\ 0 & r \geq 2^{1 / 6} \sigma_{i j}\end{cases}
$$




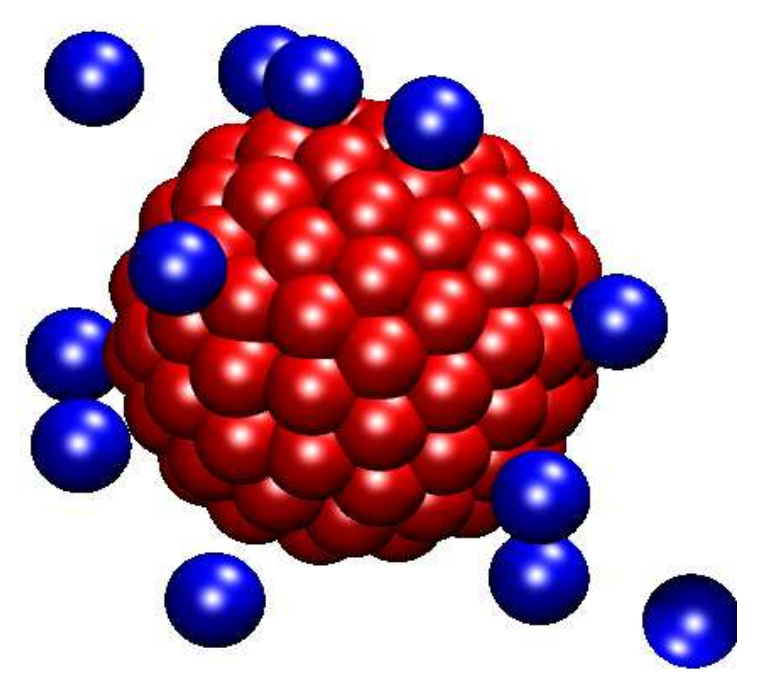

Figure 1. Raspberry-like model of a colloidal sphere. There is a central large bead of radius $R=3$ and charge $Z=20$. The small beads of radius 1 are connected with their nearest neighbors on the surface via FENE bonds. A repulsive soft-core potential is also operating between all the monomers. The counterions are moving freely in space and interact with the central bead via the Coulomb potential and the repulsive LJ potential.

An additional repulsive LJ bead is introduced at the center of the sphere in order to maintain its shape. In Eq. 4, $i, j$ denote either a central ("c"), or a surface ("s") bead, or an ion ("i"). The unit system is completely defined by the surface bead parameters by setting $\epsilon_{s s}, \sigma_{s s}$, and the surface bead mass $m_{s}$ to unity. All other beads have mass 1, too. The interaction between the central bead and the surface beads is described by $\sigma_{c s}=3$, which is thus the sphere radius, and $\epsilon_{c s}=48$, while the interaction with the ions is characterized via $\sigma_{c i}=4$ and $\epsilon_{c i}=1$. There are no interactions between the ions and the surface beads. We place the colloid charge at the central bead and add an appropriate number of counterions (LJ beads with $\sigma_{i i}=1, \epsilon_{i i}=1$ ) outside the sphere. The electrostatic interaction is taken into account via the Coulomb potential

$$
V_{e l}(r)=\lambda_{B} k_{B} T \frac{z_{i} z_{j}}{r}
$$

between the various charges, where the standard Ewald summation technique ${ }^{20}$ is applied, as it is appropriate for a small number of charges. In Eq. $5, \lambda_{B}=$ $e^{2} /\left(4 \pi \varepsilon_{0} \varepsilon_{r} k_{B} T\right)$ is the Bjerrum length, $k_{B}$ the Boltzmann constant, $z_{i}$ the valency of species $i$ in units of the elementary charge $e$, and $T$ the temperature. The Bjerrum length in all our runs was set to $\lambda_{B}=1.3$. This value is motivated by an attempt to mimic an aqueous dispersion of spherical sodium dodecyl sulfate micelles, which in reality have radius of $2 \mathrm{~nm}$ and carry 60 elementary charges. ${ }^{21,22}$

The LB lattice constant is chosen as one (in our LJ unit system), and the fluid is simulated in a cubic box with periodic boundary conditions and box size $L=30$, which 


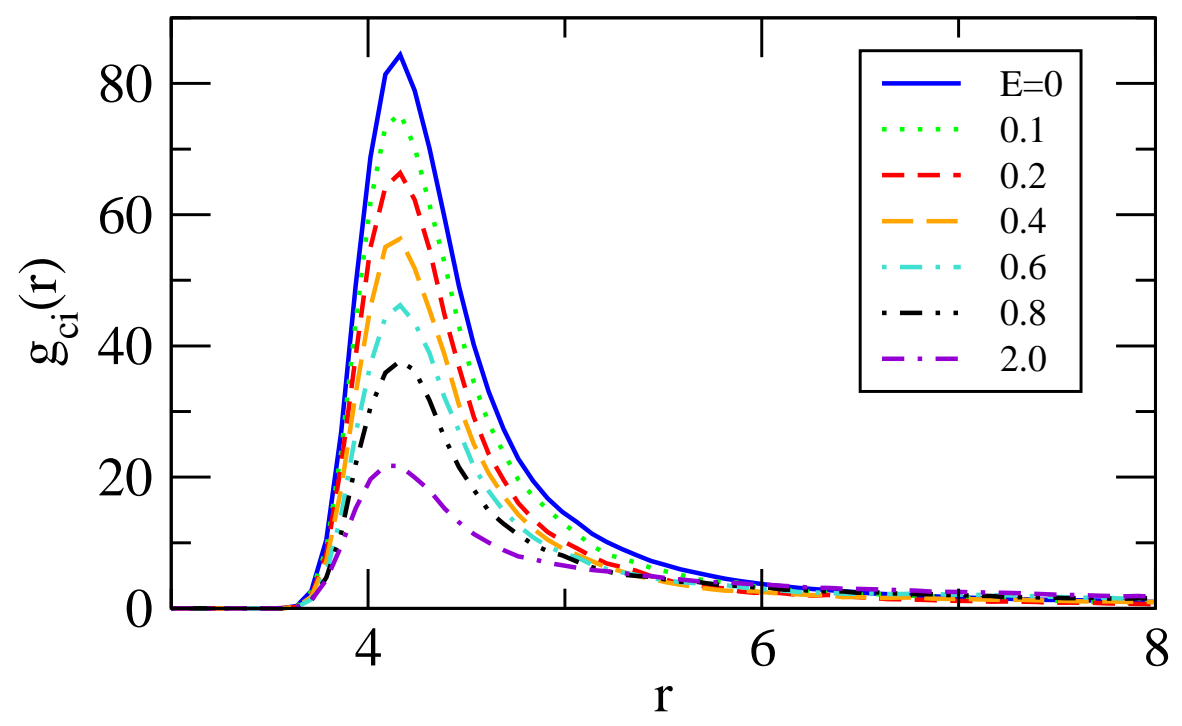

Figure 2. Colloid-ion radial distribution functions for a colloidal particle of charge -60 in an external electric field $E$.

defines the effective finite concentration of our system; this corresponds to a volume fraction of about $1 \%$ if $\sigma_{c i}$ is taken as the particle radius. The force acting on the surface beads (or ions) is given by

$$
\vec{F}=-\Gamma(\vec{V}-\vec{u})+\vec{f} .
$$

Here, $\Gamma$ is the "bare" 19 friction coefficient, $\vec{V}$ and $\vec{u}$ are the velocities of the bead and the fluid (at the position of the bead), respectively, while $\vec{f}$ is a Gaussian white noise force with zero mean, whose strength is given via the standard fluctuation-dissipation theorem ${ }^{18,19}$ to keep the surface beads and ions at the same temperature as the solvent. In our simulation we used a friction constant $\Gamma=20$, a temperature $k_{B} T=1$, a fluid mass density $\rho=0.85$, and a kinematic viscosity $\nu=3$, resulting in a dynamic viscosity $\eta=2.55$. At least $20000 \mathrm{MD}$ steps were performed to equilibrate the initial random bead configuration before the interaction with the LB solvent was turned on. A multiple time step technique was used, with the MD time step of $0.005 \mathrm{LJ}$ time units and LB field update interval of 0.01 . Further details on the method can be found in. ${ }^{16,18,19}$

We should note that the application of periodic boundary conditions makes the electrophoresis in our system somewhat different from the experimental case where a multi-colloid system reacts to the external field. The particle is both hydrodynamically and electrostatically coupled to its periodic images. Therefore, we measure in fact the mobility of a periodic particle array arranged in a simple cubic lattice with lattice spacing of the simulation box size. 


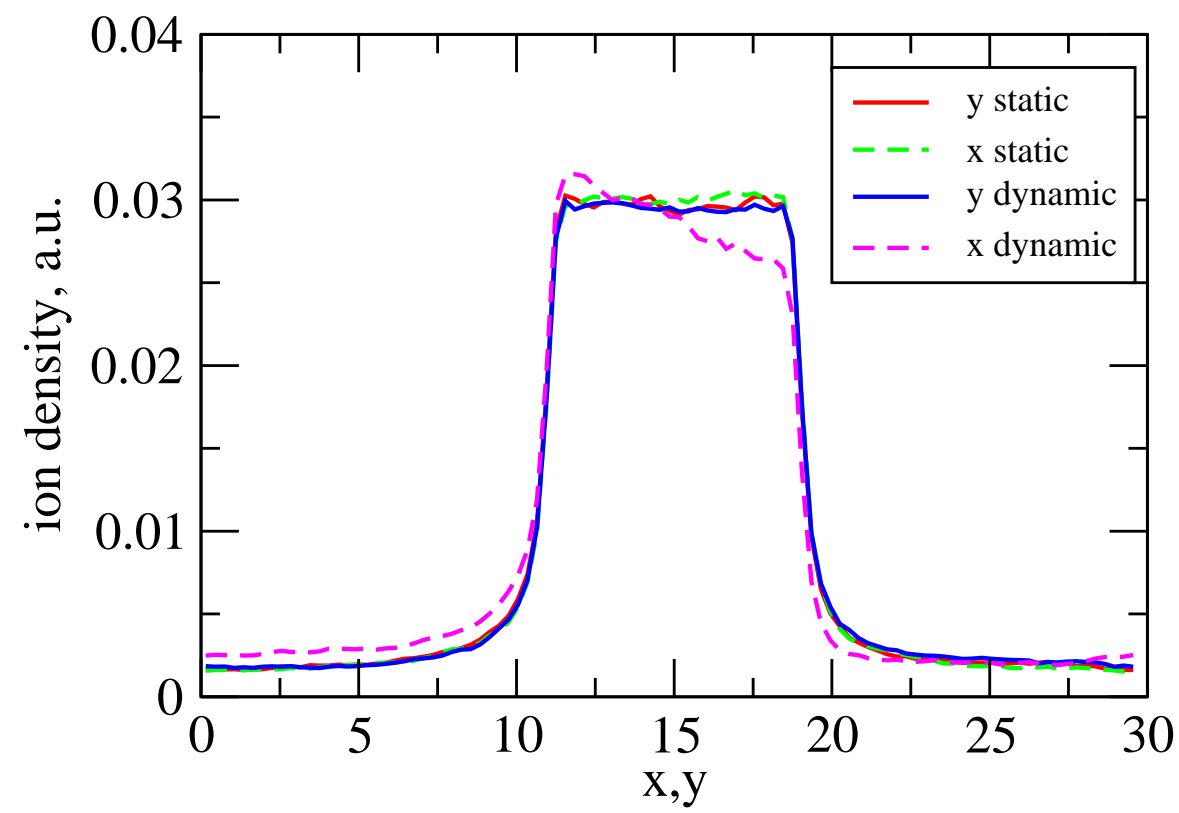

Figure 3. Ion number density profile for a colloid of charge -120 (i) in static conditions (solid lines) and (ii) in an external electric field of absolute value $E=0.2$ so that the particle moves in positive $x$ direction (dashed lines). The density profiles are taken in $x$ direction (along the field, $y$ and $z$ dependence integrated out) and in $y$ direction (perpendicular to the field, $x$ and $z$ dependence integrated out). The peak on the left hand side indicates ion accumulation behind the particle.

\section{Results}

We first investigated the character of the particle motion and the ionic density distribution in a constant homogeneous electric field. We studied a particle of charge -60 with 60 counterions. No salt ions were added, i. e. the screening layer consisted of counterions only. Several field values were considered, beginning in the regime in which the drift was barely detectable, and ending in the regime where the mean drift velocity was of order unity in our LJ units. In all cases, a steady motion with fairly constant velocity (except for thermal fluctuations) was reached within a few LJ time units. In Fig. 2 one can see the spherically averaged stationary state radial distribution functions (rdf's) for the colloid-ion correlation for several field values. The rdf's are qualitatively similar for the static and the dynamic case; in essence, only the peak height of the distribution is affected by the external field. The main peak of the colloid-ion rdf decreases substantially upon increasing the external field. This effect can be explained by (i) a stronger electric force acting on the ions and (ii) faster motion of the colloid with its ionic cloud and hence stronger friction, which in combination result in stripping an additional fraction of the double layer. It is important to note that this latter regime is already beyond the limits of validity of the standard electrokinetic theory, which is based on linear response treatment of the external field effects. 

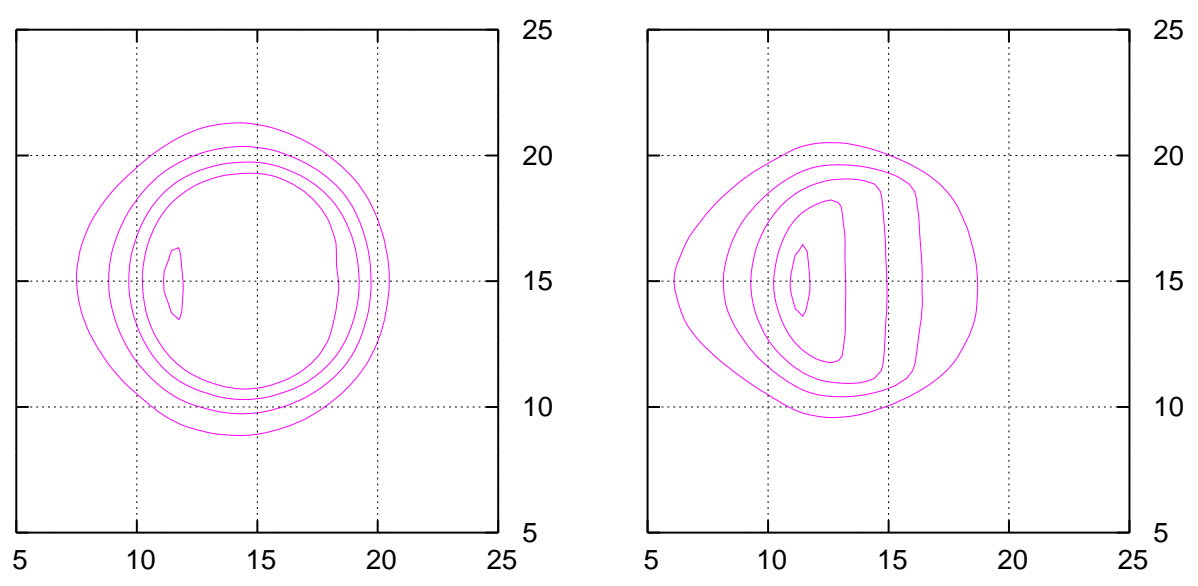

Figure 4. Contour plots of the ion density distribution for a colloid of charge -60 in an external electric field $E=0.2$ (left) and $E=0.6$ (right). The particle moves in the positive $x$ direction and its center is located at $[15,15]$, while $\sigma_{c i}=4$. For this plot, we have only retained the $x$ and $y$ dependence of the density, while the $z$ dependence has been integrated out. The isolines are stretched to the left hand side, which indicates ion accumulation behind the particle.

In a stationary state, the motion of the ions in the double layer is controlled by a balance of the external homogeneous force field, the attraction to the colloid surface, the ion-ion interaction, and the friction force. Thus, the stationary state double layer around the colloid is in general asymmetric. The density profile along the field (Fig. 3, dashed curves) does not coincide any longer with that taken across the field direction (solid curves). One can notice an accumulation of ions behind the particle (the particle is moving towards larger $x$ ) and a rarefied region just in front of the particle. Note however that the relative difference is not large, which means that the ionic cloud is not strongly polarized. Contour plots of the ionic distribution for a colloid with $Z=-60$, $E=0.2$ and $E=0.6$ are shown in Fig. 4. The asymmetry of the ionic cloud is not very large for these fields, which allows us to regard the ionic cloud as spherical, for example in determining the effective charges and potentials in a simple way.

In order to define a dynamic effective charge of the colloid, which is related to the zeta potential, we look at the radial distribution of the ionic velocity in the double layer, taking the component in the direction of the external field. As the instant velocities are governed by thermal fluctuations and thus do not carry a sufficient amount of the interesting information, we calculated the average velocity over $10 \mathrm{LJ}$ time units. This procedure averages out the stochastic components of the velocity so that the directional drift dominates the motion. The corresponding average curves are shown in Fig. 5. The velocity is shown relative to the mean velocity of the colloid. We see that the ions in the nearest surroundings of the particle surface move along with the colloid. 

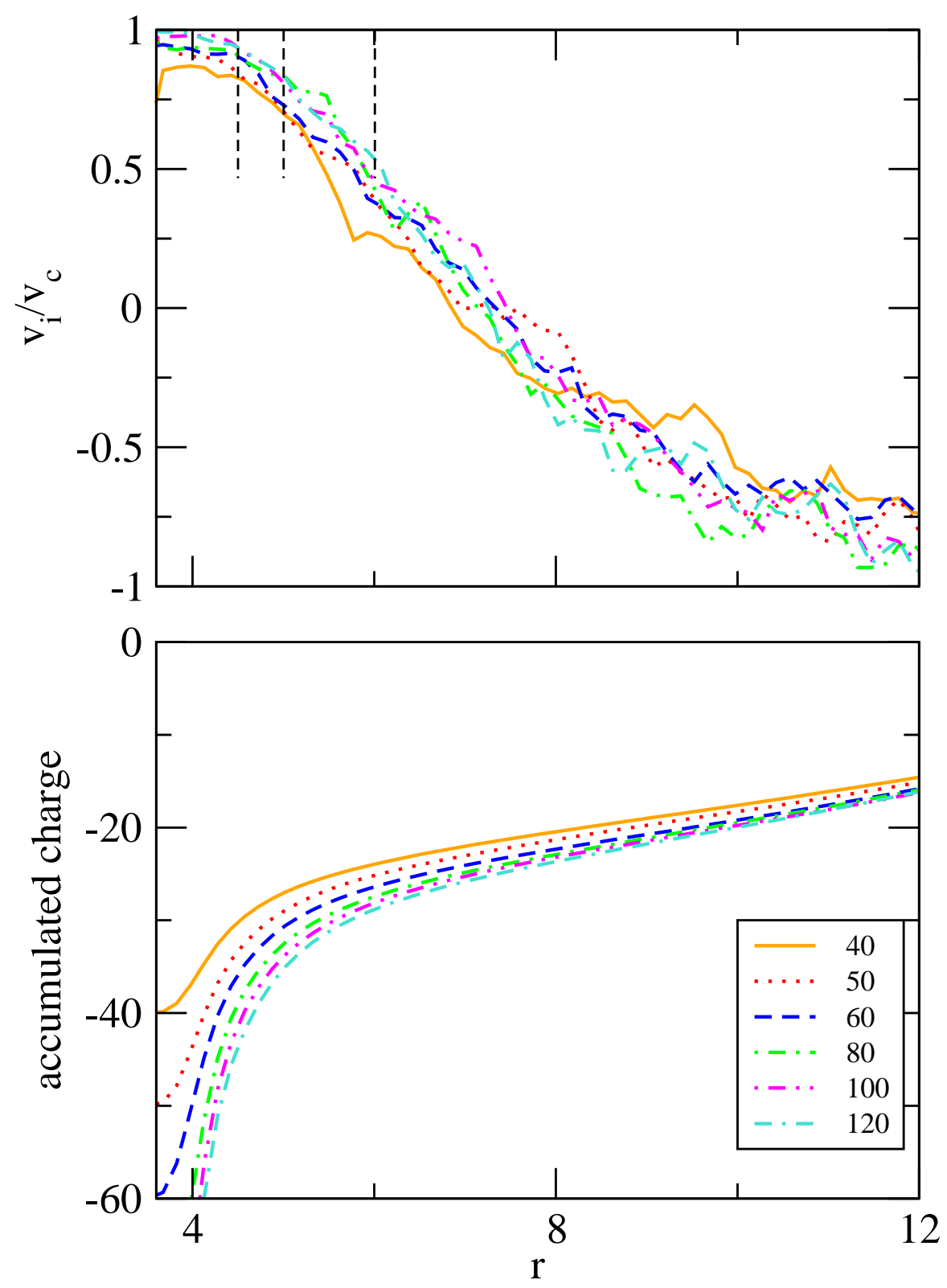

Figure 5. Top: Radial distribution of the ionic drift velocity along the external field direction for colloids of different charge in an external electric field $E=0.2$. The thin dashed lines indicate the three supposed positions of the slip surface. Bottom: Integrated charge curves for the same samples. The curves in both plots are marked by the bare colloid charge. 


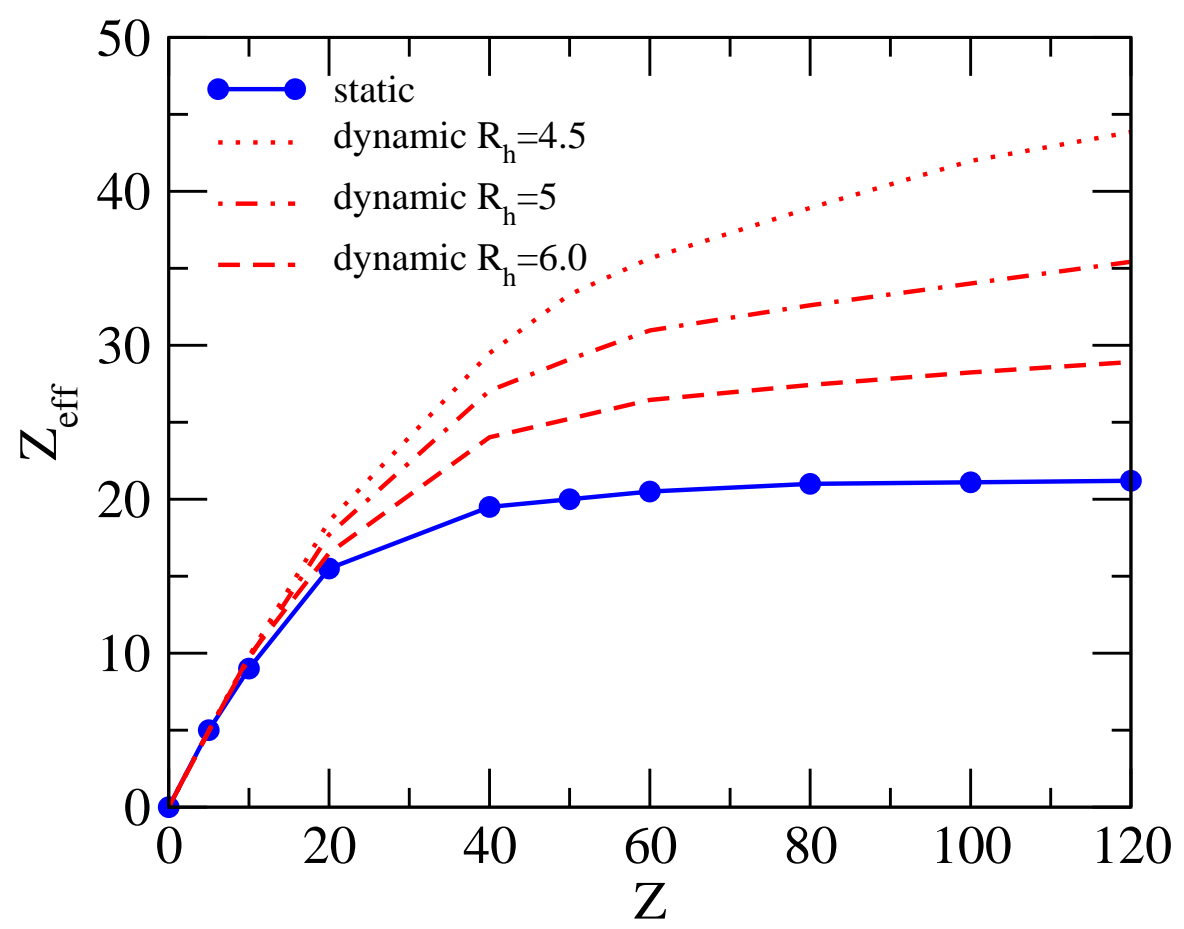

Figure 6. Static and dynamic effective charge of a colloidal particle as a function of its structural charge. The static charge is calculated using a static simulation and the inflection point criterion. The dynamic charge is found using integration of the stationary charge distribution within the assumed shear surface at $E=0.2$. The corresponding colloid volume fraction is about $1 \%$.

The relative velocity reaches unity at about $r=4$, which agrees with the peak of the ionic radial distribution. From there, the correlation decreases monotonously and turns negative at about $r=8$. We then see a region of anti-correlation spanning until $r=15$, the half-box distance. In this region, the ionic drift velocity is anti-parallel to the colloidal one. Since there is no well-defined plateau near the particle, it is not possible to define a slip surface (where the ion motion stops to be fully correlated with that of the central particle) unambiguously, and thus we have taken several reasonable values for the corresponding radius $(r=4.5, r=5.0, r=6.0)$. This defines, on the one hand, the effective hydrodynamic radius of the overall object, and on the other hand the effective charge (i. e. the charge within the slip surface). Furthermore, the zeta potential would be the electrostatic potential at this surface.

We now turn to the relation between the static and dynamic effective charges of a colloidal particle. This question reflects one of the most common problems for technological applications of colloidal dispersions: Predicting the stability of a suspension based on electrophoretic mobility data or vice versa. Extensive studies have been dedicated to the interpretation of electrokinetic data in order to use them for particle characterization. ${ }^{3,4}$ The discrepancy between the two types of effective charges has a quite obvious origin: The static and dynamic experimental setups probe different 
properties of the ionic double layer. While static properties like structure factor, elastic moduli, phase diagrams are affected by the long-range decay of the potential, the particle mobility depends primarily on the resistance of the double layer to the viscous drag force. In both cases, it is not the particle surface charge (or bare charge) that enters the definitions of the relevant electrostatic and electrokinetic potentials but some effective value, which is usually lower than the bare charge. Normally, it is difficult to access both the static and the dynamic properties of the same system and such a work requires a special effort. Recently, a comparison between the static and dynamic effective charges calculated from crystal shear moduli and suspension conductivity, respectively, was done. $^{23}$ The dynamic effective charge was found to be $40 \%$ larger than the static one for several sets of silica and latex particles in law-salt conditions. We expect that our system without added salt shows similar features as the hydrodynamic coupling of the ions to the particle is a very short-range effect.

The colloid-ion rdf's were integrated at vanishing external field to obtain accumulated charge curves and to determine the static effective charge by means of the inflection point criterion. ${ }^{24,25}$ Since it is known that most properties (in particular, the structure factor) are not particularly sensitive to the precise definition of the static charge (several different criteria turn out to be roughly equivalent), ${ }^{24}$ we have just taken this for convenience. For charges smaller that 20, no inflection point could be detected and we took the effective charge at the inflection point location of the more strongly charged particles. In the dynamic case, the external field $E=0.2$ was applied. The radial charge distributions were integrated in the stationary state, however not up to the inflection point but rather up to the supposed slip surface, taking the three different values $r=4.5,5.0$, and 6.0 (see discussion above, and Fig. 5)

The resulting charge curves are shown in Fig. 6. Both the static and dynamic effective charge grow linearly at $Z \rightarrow 0$ and more slowly at $Z>40$. The static charge curve saturates at a value of $Z_{\text {eff }}^{s} \approx 22$, while the dynamic charges continue to increase. The charge calculated with the largest slip surface radius $r=6.0$ reaches a magnitude of about 29, which is $30 \%$ higher than the static charge value. The two others, $r=4.5$ and $r=5.0$, stop at 40.5 and 35.5, respectively. The observed difference between the static and dynamic charges is by no means surprising as the reasonable radii of the slip surface are all smaller than the inflection point position, $r \approx 8$, and hence include less counterions. Let us recall that the correlation between the colloid and ion velocity in the dynamic measurement vanishes at this distance $r=8$ (see Fig. 5). In our case of no salt the relation between the static and dynamic charges is indeed similar to what has been reported for latex and silica spheres, ${ }^{23}$ although we measured the static and dynamic charges in a different way. We should note that this ratio is obviously not universal and should hold only for electrokinetically similar systems. We expect it to be much closer to unity in strongly screened systems with high salt content as the double layer thickness would be smaller and thus closer to the slip surface.

We also looked at the particle electrophoretic mobility as a function of particle bare charge. The considered charges range from 0 to 120 . The electric field was set to 


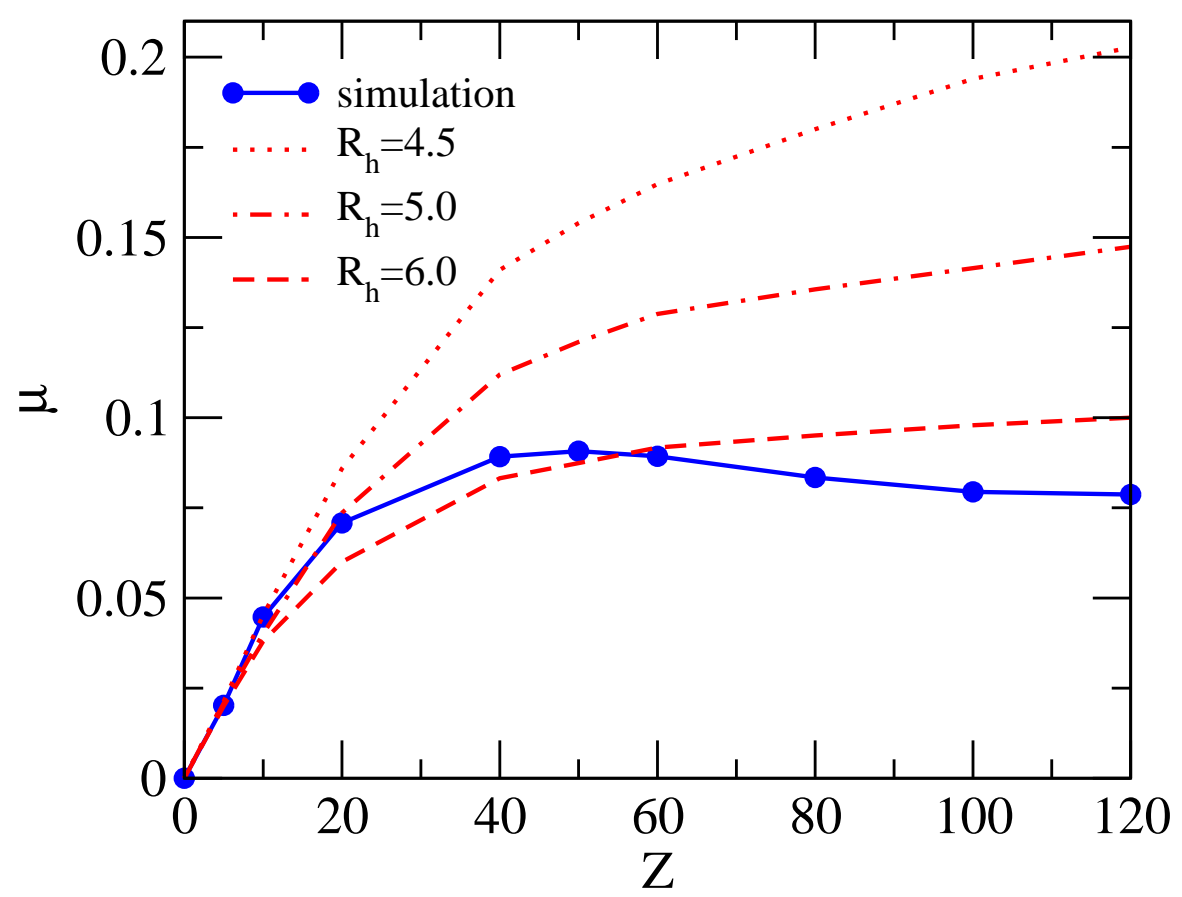

Figure 7. Electrophoretic mobility of a particle of charge $Z$ and radius $R=3$ at external field $E=0.2$ in a simulation box of length 30 as obtained directly from simulation (solid curve) or calculated from Eq. 2 with the indicated slip surface position and $f(\kappa R)=1: \mu=Z_{e f f}^{d} e /\left(6 \pi \eta R_{h}\right)$.

a compromise value of 0.2 to make the particle drift pronounced but to avoid strong asymmetry of the double layer (see Fig. 4). The result is displayed in Fig. 7. At low charges $Z \leq 10$, the mobility increases with the charge almost linearly. This behavior can be readily explained by a plain increase of the net force acting on the particle. At very low charge, the interaction between the colloid and the ions does not exceed $k_{B} T$, which makes the ion distribution only slightly correlated with the colloid. At higher charges however the increase of the electrophoretic mobility is slowing down, marking the onset of ion condensation on the colloid surface. At $Z \approx 50$, the mobility shows a maximum. Finally, at $Z>60$, we see a slight decrease in the mobility upon further charge increase. This behavior can be explained by the combined effect of colloid charge saturation and increase of the friction due to the more and more packed double layer. A similar behavior was reported already back in seventies for charged emulsion droplets. ${ }^{3}$ A calculation using Eq. 2 with the preselected fixed slip surface positions and $f(\kappa R)=1$ fails to reproduce this feature. The calculated mobilities shown in Fig. 7 (dashed or dotted curves) grow monotonically. One can see however that the curve with the smaller $R_{h}=4.5$ describes better the initial part of the measured mobility curve while the final part is described better by $R_{h}=6$. Obviously, a consistent description requires that the position of the slip surface (the hydrodynamic radius) shifts to larger distances with increasing bare particle charge. 


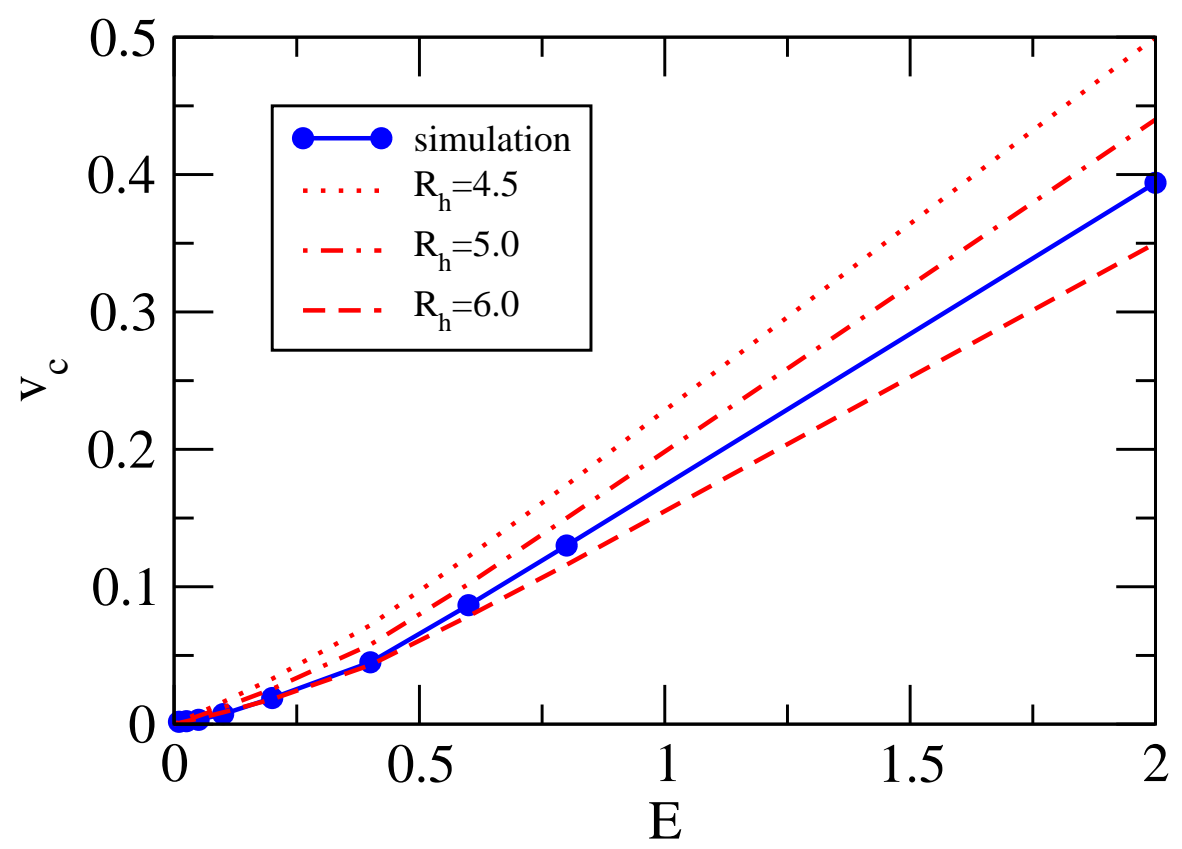

Figure 8. Stationary drift velocity of a colloidal particle with charge -60 in an external electric field $E$ as obtained from simulation or calculated using Eq. 1-2 with the indicated slip surface position and $f(\kappa R)=1: v=Z_{\text {eff }}^{d} e E /\left(6 \pi \eta R_{h}\right)$

We finally looked at the colloid velocity as a function of the external field (Fig. 8). In this way one can estimate the limits of validity of linear response theory. In a weak field up to $E \approx 0.5$, the velocity grows linearly (solid line) as expected for a system with a constant electrophoretic mobility. In a stronger field however a faster growth is seen, which means that the mobility increases. We again supposed the three different slip surface positions to calculate the velocity. A calculation with the shortest distance $r=4.5$ predicts a too high velocity for all field values. The curves corresponding to $r=5$ and $r=6$ bracket the velocity values obtained by direct measurement in the simulation. The best prediction can hence be made with $r \approx 5.5$. The most important observations in this test are as follows: We found that for large field values $E>0.5$ no pronounced correlation of the ion velocity with the colloid velocity was observed. The ratio $v_{i} / v_{c}$ did not exceed 0.4 for $E=2$. Thus, the slip surface could not be defined at all. This fact is supposedly related to the distortion of the ionic cloud, which was illustrated in Fig. 4. One should remember that the slip surface has to be defined by the ionic distributions in the limit $E \rightarrow 0$. Nevertheless, a reasonable estimate of the drift velocity can be obtained using the slip surface position found in the linear regime at small but finite $E$. 


\section{Summary}

We applied a hybrid MD/LB simulation method for studying colloidal electrophoresis. A combination of the primitive electrolyte model with accurate treatment of the hydrodynamics allows us to access experimentally measurable quantities like the electrophoretic mobility and the underlying double layer structure at the same time. Our simulation model shows the non-trivial coupling between charge distribution and hydrodynamic flow, and the corresponding non-linear effects. By analyzing the stationary ionic velocity distribution around the colloid, we attempted to find the position of the slip surface and the corresponding dynamic effective charge. Obviously, the data for our model do not permit a unique definition; nevertheless, from a combined analysis of the distribution, and the particle mobility in terms of the Hückel theory, we could find reasonable values. The dynamic charge appears to be somewhat larger than the static effective charge estimated from the inflection point criterion, which agrees with experimental findings for charged silica and latex colloidal spheres. Our statistical definition of the slip surface combined with the Hückel theory of electrophoresis gives a reasonable description of the particle mobility in weak electric fields in a salt-free suspension. A more detailed comparison with existing theories as well as with multicolloid simulations will (hopefully) provide a deeper understanding of these issues in the future.

\section{Acknowledgments}

We thank Jürgen Horbach, Olga Vinogradova, and Kurt Kremer for stimulating discussions. This work was funded by the SFB TR 6 of the Deutsche Forschungsgemeinschaft.

\section{References}




\section{References}

${ }^{1}$ Smoluchowksi M von 1903 Bull. Akad. Sci. Cracovie, Classe Sci. Math. Natur. 1182 ${ }^{2}$ Hückel E 1924 Physik. Z. 25204

${ }^{3}$ Dukhin S S and Deryaguin B V 1974 Electro-kinetic phenomena in Surface and Colloid Science vol. 7 (Matijevic E ed.) (New York: John Wiley)

${ }^{4}$ Hunter R J 1981 Zeta potential in colloid science (London: Academic Press)

${ }^{5}$ Attard P, Antelmi D, and Larson I 2000 Langmuir 161542

${ }^{6}$ Henry D C 1931 Proc. R. Soc. (London) Ser. A 133106

${ }^{7}$ Wiersema P, Loeb A, Overbeek J 1966 J. Colloid Interface Sci. 2278

${ }^{8}$ O'Brien R and White L 1978 J. Chem. Soc. Faraday Trans. 741607

${ }^{9}$ Lozada-Cassou M and Gonzáles-Tovar E 2001 J. Colloid Interface Sci. 239285

${ }^{10}$ Tanaka M and Grosberg A Y 2001 J. Chem. Phys 115567

${ }^{11}$ Ladd A J C 1993 Phys. Rev. Lett. 701339

${ }^{12}$ Ladd A J C, Hu Gang, Zhu J X, and Weitz D A 1995 Phys. Rev. E 526550

${ }^{13}$ Hagen M H J, Frenkel D, and Lowe C P 1999 Physica A 272376

${ }^{14}$ Horbach J and Frenkel D 2001 Phys. Rev. E 64 061507-1

${ }^{15}$ Horbach J Private communication

${ }^{16}$ Lobaskin V and Dünweg B 2004 New J. Phys. 654

${ }^{17}$ Ladd A J C 1994 J. Fluid Mech. 271285

${ }^{18}$ Ahlrichs P and Dünweg B 1998 Int. J. Mod. Phys. C 91429

${ }^{19}$ Ahlrichs P and Dünweg B 1999 J. Chem. Phys. 1118225

${ }^{20}$ Allen M and Tildesley D J 1987 Computer simulation of liquids (Oxford: Oxford University Press)

${ }^{21}$ Lobaskin V and Linse P 1999 J. Chem. Phys. 1114300

${ }^{22}$ Lobaskin V, Lyubartsev A, and Linse P 2001 Phys. Rev. E 63020401

${ }^{23}$ Wette P, Schöpe H J, and Palberg T 2002 J. Chem. Phys. 11610981

${ }^{24}$ Belloni L 1998 Colloids and Surfaces A 140227

${ }^{25}$ Deserno M, Holm C, and May S 2000 Macromolecules 33199 